\title{
ON THE HOPF INDEX THEOREM AND THE HOPF INVARIANT ${ }^{1}$
}

\author{
BY KUO-TSAI CHEN
}

Let $f: N \rightarrow M$ be a $C^{\infty}$ map of oriented compact manifolds, and let $L$ be an oriented closed submanifold of codimension $q \geqslant 1$ in $M$. If $w$ is a closed form Poincare dual to $L$, we show that $f^{-1} L$, with multiplicities counted, is Poincaré dual to $f^{*} w$ in $N$ and is even meaningful on a "secondary" level. This leads to generalized versions of the Hopf invariant, the Hopf index theorem and the Bezout theorem.

We assume that the connected components $\Gamma_{1}, \ldots, \Gamma_{l}$ of $f^{-1} L$ are submanifolds of codimension $q$ in $N$. Let ord $\Gamma_{i}$ be the intersection number of $L$ and $f \mid B$, where $B$ is a $q$-dimensional submanifold meeting $\Gamma_{i}$ transversally at a single point. A proper choice of orientations makes ord $\Gamma_{i} \geqslant 0$.

\section{Theorem 1. The cycle $\Sigma\left(\right.$ ord $\left.\Gamma_{i}\right) \Gamma_{i}$ is Poincare dual to $f^{*} w^{2}$}

This assertion improves a known theorem, which requires that $f$ is transversal to $L$ and, consequently, ord $\Gamma_{i}=1$.

THEOREM 2. Let $w^{\prime}$ be an integral closed $q^{\prime}$-form on $M$ with $q+q^{\prime}-1$ $>\operatorname{dim} M$. If both $f^{*} w$ and $f^{*} w^{\prime}$ are exact with $f^{*} w^{\prime}=d u$ on $N$ and if $\sigma$ is a closed $p$-form on $N$ with $p+q+q^{\prime}=\operatorname{dim} N$, then

$$
\int_{N} f * w \wedge u \wedge \sigma=\sum\left(\text { ord } \Gamma_{i}\right) \int_{\Gamma_{i}} u \wedge \sigma
$$

CoRollary. Let $f: N \rightarrow M$ be an arbitrary $C^{\infty}$ map (without any condition on $\left.f^{-1} L\right)$. If $r$ is the least positive integer making $r H_{q+q^{\prime}-1}(N ; Z)$ free abelian, then the cohomology class of $r f^{*} w \wedge u$ is integral.

A sketched proof of Theorem 1 runs as follows. There exists a $(q-1)$ form $v$ on $M-L$ with $d v=w \mid M-L$. Let $\sigma$ be a closed $p$-form on $N$ such that

\footnotetext{
Received by the editors January 24, 1981.

1980 Mathematics Subject Classification. Primary 57R20, 55Q25; Secondary 55M05, $14 \mathrm{C} 99$.

1 Work supported in part by NSF MCS 79-00321.

2 The author wishes to thank the referee for pointing out the general validity of Theorems 1 and 2. Our proof of these theorems is a modification of the original version, which, however, already suffices to cover Theorem 3 and other applications in this note.
} 
$p+q=\operatorname{dim} N$. Then, on $N-\Gamma, d\left(f^{*} v \wedge \sigma\right)=f^{*} w \wedge \sigma$. Let $T_{i}(\epsilon)$ be the $\epsilon$ tubular neighborhood about $\Gamma_{i}$. Then

$$
\int_{N} f^{*} w \wedge \sigma=-\sum \lim _{\epsilon \rightarrow 0} \int_{\partial T_{i}(\epsilon)} f^{*} v \wedge \sigma
$$

The theorem will follow from the formula

$$
\lim _{\epsilon \rightarrow 0} \int_{\partial T_{i}(\epsilon)} f^{*} v \wedge \sigma=-\left(\text { ord } \Gamma_{i}\right) \Gamma_{i} \sigma .
$$

This formula is verified by using, in essence, the approach described below. The integration over $\partial T_{i}(\epsilon)$ is first performed over fibers and then over $\Gamma_{i}$. Since $\sigma$ is defined everywhere on $N$, its contribution during the integration over fibers can be ignored as $\epsilon \rightarrow 0$. The fiber of the $\epsilon$-tubular neighborhood $T_{i}(\epsilon)$ is a $q$ dimensional submanifold $B(\epsilon)$ transversal to $\Gamma_{i}$. We show that ord $\Gamma_{i}=$ $-\lim _{\epsilon \rightarrow 0} \int_{\partial B(\epsilon)} f * v$ and thus (1).

In order to prove Theorem 2, we take note that $v \wedge w^{\prime}=0$ on $M-L$ and that $d\left(f^{*} v \wedge u\right)=f^{*} w \wedge u$ on $N-\Gamma$. The theorem follows from the Stokes theorem and the formula

$$
\lim _{\epsilon \rightarrow 0} \int_{\partial T_{i}(\epsilon)} f^{*} v \wedge u \wedge \sigma=-\left(\text { ord } \Gamma_{i}\right) \int_{\Gamma_{i}} u \wedge \sigma .
$$

Realizing that $w^{\prime}$ can be modified so that $w^{\prime}$ vanishes on a neighborhood of $L$, we can verify this formula in the same way as for (1).

EXAMPLE 1. Let $M$ be the $q$-sphere bundle obtained by compactifying a $C^{\infty}$ vector bundle of fiber dimension $q$ over an oriented compact manifold of dimension $<q-1$. Let $L_{0}$ and $L$ be respectively the zero and the "infinity" sections of $M$. Let $f: N=S^{2 q-1} \rightarrow M$ be a $C^{\infty}$ map such that both $f^{-1} L_{0}$ and $f^{-1} L$ are smooth and of codimension $q$ in $N$. Let $f^{*} w=d u$ and let $\Sigma\left(\right.$ ord $\left.\Gamma_{i}\right) \Gamma_{i}=\partial \mathrm{Z}$ in $N$. According to Theorem 2,

$$
\begin{aligned}
\int_{S^{2 q-1}} f^{*} w \wedge u & =\int_{\mathrm{Z}} f^{*} w \\
& =\text { the algebraic linking number of } f^{-1} L \text { and } f^{-1} L_{0}
\end{aligned}
$$

The Whitehead integral formula for the Hopf invariant [3] is thus valid in this generalized situation.

ExAmple 2. Let $M$ be the fiber bundle obtained from a $C^{\infty}$ complex (or quaternion) vector bundle by replacing each fiber with its complex (or quaternion) projective compactification. Let $L_{0}$ be the zero section and let $L$ be the union of the hyperplanes at infinity of the fibers. Theorem 1 implies that, under reasonable conditions, the homology class of the poles of a $C^{\infty}$ section of $M$ does not depend on the choice of the section. 
EXAMPLE 3. Let $M=G\left(k, C^{n}\right)$ be the Grassmannian of complex $k$-planes in $C^{n}, n>k$. Let $L=G\left(k, C^{n-1}\right)$. Then the $k$ th Chern form $w$ of $M$ is Poincare dual to $L$. Set $w^{\prime}=w^{n-k}$. It can be verified that, if $N$ is the associated $(2 k-1)$-sphere bundle of the universal vector bundle of $M$ and if $f: N \rightarrow M$ is the bundle map, then $f^{*} w^{\prime}=d u$ and $\int_{N} f^{*} w \wedge u=1$.

Let $E \rightarrow N$ be a $C^{\infty}$ complex $k$-plane bundle and let $s: N \rightarrow E$ be a $C^{\infty}$ section having $\Delta$ as its zero set whose connected components are $\Delta_{1}, \ldots, \Delta_{l}$. We assume that each $\Delta_{i}$ is smooth and of codimension $2 k$ in $N$. The index of $s$ along $\Delta_{i}$ is the integer index $\Delta_{i}$, which is the usual index of the restriction of $s$ to a $2 k$-dimensional submanifold meeting $\Delta_{i}$ transversally at a single point under a suitable choice of orientations. Theorem 1 leads to a generalized Hopf index theorem for complex vector bundles.

Theorem 3. The homology class of $\Sigma\left(\right.$ index $\left._{s} \Delta_{i}\right) \Delta_{i}$ is Poincaré dual to the kth Chern class of $E$.

The main feature of this result is that the classical notion of index continues to provide multiplicities of the zero set $\Delta$. Under certain generic conditions, there is a theorem of Griffiths [2] (see also [3, p. 413]) on Poincare duals of all Chern classes of $E$. Theorem 3 extends a part of Griffiths' theorem to zero-sets with multiplicities.

Using Theorem 3, we point out a proof of a generalized version of the classical Bezout theorem. Let $z=\left(z_{1}, \ldots, z_{n}\right)$ be the coordinates of $C^{n}$, and let $g_{1}(z), \ldots, g_{r}(z)$ be polynomials of respective degrees $d_{1}, \ldots, d_{r}, r \leqslant n$. We assume that the common zero set $\Delta$ of $g_{1}, \ldots, g_{r}$ in $C P^{n}$ is smooth and of pure codimension $r$. Let $H$ be the hyperplane line bundle of $C P^{n}$. Then $\left(g_{1}, \ldots, g_{r}\right)$ is a section of $H^{d_{1}} \oplus \cdots \oplus H^{d_{r}}$, and so is $\left(z_{1}^{d_{1}}, \ldots, z_{r}^{d_{r}}\right)$. Hence, with multiplicity counted, $\Delta$ must be homologous to $d_{1} \ldots d_{r}\left(C P^{n-r}\right)$.

\section{BIBLIOGRAPHY}

1. P. A. Griffiths, Hermitian differential geometry, Chern classes, and positive vector bundles, Global Analysis (Papers in honor of K. Kodaira), Univ. of Tokyo Press, Tokyo and Princeton Univ. Press, Princeton, N. J., 1969, pp. 185-251.

2. P. A. Griffiths and J. Harris, Principles of algebraic geometry, Wiley, New York, 1978.

3. H. Whitney, Geometry integration theory, Princeton Univ. Press, Princeton, N.J., 1957.

DEPARTMENT OF MATHEMATICS, UNIVERSITY OF ILLINOIS, URBANA, ILLINOIS 61801 
